\section{P137 DE NOVO LUPUS NEPHRITIS DURING BELIMUMAB TREATMENT}

${ }^{1}$ Ioannis Parodis, ${ }^{2}$ Edward M Vital, ${ }^{3}$ Andreas Jönsen, ${ }^{2}$ Sabih-Ul Hassan, ${ }^{4}$ Dag Leonard, ${ }^{5}$ Per Eriksson, ${ }^{3}$ Anders A Bengtsson, ${ }^{1}$ Iva Gunnarsson, ${ }^{4}$ Lars Rönnblom, ${ }^{5}$ Christopher Sjöwall. ${ }^{1}$ Karolinska Institute and Karolinska University Hospital, Stockholm, Sweden; ${ }^{2}$ University of Leeds, Leeds, UK; ${ }^{3}$ Lund University, Lund; ${ }^{4}$ Uppsala University, Uppsala; ${ }^{5}$ Linköping University, Linköping, Sweden

\subsection{6/lupus-2020-eurolupus. 181}

Background Standard-of-care (SoC) for systemic lupus erythematosus (SLE) includes use of glucocorticoids, antimalarials, immunosuppressive agents and biologics. Selection of drug(s) for each unique patient is mainly based on the severity of afflicted organ system(s), the global disease activity, and serological status (i.e. anti-dsDNA and complement levels). Development of lupus nephritis (LN) during treatment with belimumab, an inhibitor of soluble $B$ cell activating factor (BAFF), has previously been reported. ${ }^{1,2}$ This observational follow-up study, utilising data from several university centers, aimed to evaluate the real-life frequency of LN during belimumab treatment.

Methods At the Swedish university centers in Linköping, Lund, Stockholm and Uppsala, as well as in Leeds, UK, all patients who received belimumab from 2011 until 2018 ( $\mathrm{N}=95 ; 52 \%$ anti-dsDNA positive, 60\% low complement) were followed longitudinally in regional observational study programmes (median follow up: 13 months). In cases of a suspected incident LN or LN relapse/worsening, renal biopsy was performed. As a comparator, non-renal SLE cases not exposed to belimumab ( $\mathrm{N}=107 ; 40 \%$ anti-dsDNA positive, $47 \%$ low complement) were encompassed; these patients were followed for a median time of 132 months and incident LN cases were registered. Total follow-up and time to LN was calculated for each group and was utilised in Cox regression analysis.
Results We observed 6/66 cases (9.1\%) of biopsy-proven de novo LN (4/6 were class III or IV) in the non-renal SLE belimumab-treated group after a median follow-up time of 7.4 (IQR: 2.7-22.2) months, and 2/29 cases (6.9\%) of LN relapse after 1 and 9 months in the belimumab-treated group with historical but quiescent $\mathrm{LN}$ at treatment initiation. In the comparator group, $2 / 107$ cases $(1.9 \%)$ of de novo LN (1 class III, 1 class IV) were observed after 21 and 50 months. Cox regression analysis revealed increased risk and/or shorter time to de novo LN in non-renal patients who received belimumab compared with non-renal SLE comparators (HR: 17.4; 95\% CI: 2.7-110.8; P=0.002). A Kaplan-Meier graph is attached.

Conclusions This observational study indicates that addition of belimumab to SoC may not prevent $\mathrm{LN}$ among patients with ongoing non-renal SLE disease activity. Selection of cases suitable for belimumab based on serological activity could be troublesome as these patients may be at high risk of developing $\mathrm{LN}^{3}{ }^{3}$ The effects of BAFF inhibition on different lymphocyte subsets which may contribute to LN susceptibility have yet to be explored. Identification of predictors of LN development among belimumab-treated patients is merited.

\section{REFERENCES}

1. Sjöwall C, Cöster L. Belimumab may not prevent lupus nephritis in serologically active patients with ongoing non-renal disease activity. Scand I Rheumatol 2014;43:428-30.

2. Staveri C, Karokis D, Liossis SC. New onset of lupus nephritis in two patients with SLE shortly after initiation of treatment with belimumab. Semin Arthritis Rheum 2017:46:788-790.

3. van Vollenhoven RF, Petri MA, Cervera R, Roth DA, Ji BN, Kleoudis CS, et al. Belimumab in the treatment of systemic lupus erythematosus: high disease activity predictors of response. Ann Rheum Dis 2012;71:1343-49.

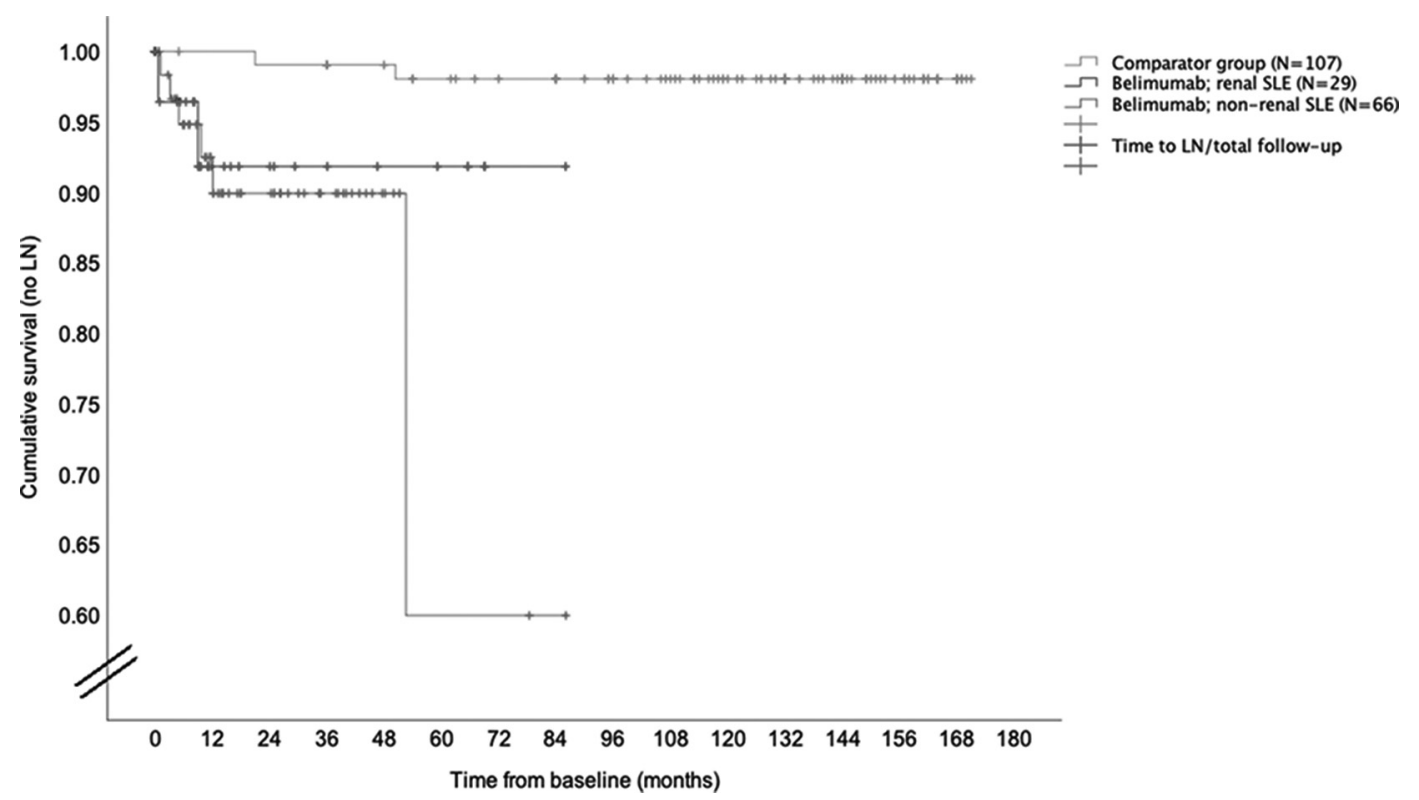

Abstract P137 Figure 1 